\title{
PERIODIC SOLUTIONS FOR A FAMILY OF EULER-LAGRANGE SYSTEMS*
}

\author{
OVIDIU CALIN ${ }^{\dagger}$, DER-CHEN CHANG ${ }^{\ddagger}$, AND STEPHEN S.-T. YAU $§$
}

Dedicated to Professor Salah Baouendi on his seventieth birthday

\begin{abstract}
In this article we study the geometry induced by the sub-Laplacian $X_{1}^{2}+X_{2}^{2}$ with $X_{1}=\partial_{x_{1}}+A_{1}(x) \partial_{t}$ and $X_{2}=\partial_{x_{2}}-A_{2}(x) \partial_{t}$. Here $A_{1}, A_{2}$ are two smooth functions defined on $\mathbb{R}^{3}$ such that $\varphi(x):=\frac{\partial A_{1}}{\partial x_{2}}+\frac{\partial A_{2}}{\partial x_{1}} \neq 0$. We first characterize necessary and sufficient conditions for horizontal curves. Then we solve the Euler-Lagrange system explicitly when $\varphi$ is linear. Moreover, we show that the solutions for the system is periodic and the Lagrange multipliers depend on the 1-connection form $\omega=d t-A_{1}(x) d x_{1}+A_{2}(x) d x_{2}$. Therefore, the arc lengths of geodesics can be computed explicitly. We also study abnormal minimizers in the last section.
\end{abstract}

Key words. subRiemannian geometry, horizontal curves, elliptic functions, Euler-Lagrange system, Jacobi's epsilon function

AMS subject classifications. Principal 53C17; Secondary 34K10, 35H20

1. Introduction and Background. Let $X_{1}, \ldots, X_{m}$ be $m$ linearly independent vector fields defined on an $n$-dimensional real manifold $\mathcal{M}$ with $m \leq n$. To induce a geometry on $\mathcal{M}$ we assume that the set of "horizontal" vector fields, or given directions, $X=\left\{X_{1}, \ldots, X_{m}\right\}$ is an orthonormal set. More precisely, let $h$ be a positive definite inner product defined on $\mathcal{D}=\operatorname{span}\left\{X_{1}, \ldots, X_{m}\right\}$ such that $h\left(X_{j}, X_{k}\right)=\delta_{j k}$ with $1 \leq j, k \leq m$. If $m=n$, this yields a Riemannian metric on $\mathcal{M}$. If $m<n$, we need further assumptions on the vector fields $\left\{X_{1}, \ldots, X_{m}\right\}$. A subRiemannian manifold $(\mathcal{M}, \mathcal{D}, h)$ is called a Heisenberg manifold if for any 1 -form $\omega$ with $\operatorname{ker}(\omega)=\mathcal{D}$, one has

$$
\operatorname{det} \omega\left(\left[X_{j}, X_{k}\right]\right)_{j k} \neq 0
$$

on $\mathcal{M}$. It is easy to see that the distribution $\mathcal{D}$ is not integrable because otherwise it would be involutive and hence $\omega\left(\left[X_{j}, X_{k}\right]\right)=0$. Hence, the equation (1.1) won't hold. The vectors $V \in \mathcal{D}_{p}$ are called horizontal vectors at $p \in \mathcal{M}$. The distribution $\mathcal{D}$ is also called horizontal distribution. The sections of the horizontal bundle $\mathcal{D}$ are called vector fields $X \in \Gamma(\mathcal{D})$. In other words, $X$ is a smooth assignment $\mathcal{M} \ni p \rightarrow X_{p} \in \mathcal{D}_{p}$. A curve $\gamma:[0, \tau] \rightarrow \mathcal{M}$ is called a horizontal curve if $\dot{\gamma}(s) \in \mathcal{D}_{\gamma(s)}$ for all $s \in[0, \tau]$, i.e.,

$$
\dot{\gamma}(s)=\sum_{j=1}^{m} a_{j}(s) X_{j}
$$

\footnotetext{
${ }^{*}$ Received May 16, 2006; accepted for publication September 8, 2006.

$\dagger$ Eastern Michigan University, Department of Mathematics, Ypsilanti, MI 48197, USA (ocalin@emunix.emich.edu). Research partially supported by a research grants from NSF \#0631541 and Eastern Michigan University.

${ }^{\ddagger}$ Georgetown University, Department of Mathematics, Washington, DC 20057-0001, USA (chang@georgetown.edu). Research partially supported by a grant from the Department of Defense and a competitive research grant at Georgetown University.

$\S$ University of Illinois at Chicago, Department of Mathematics, Statistics and Computer Science, Chicago, IL 60607-7045, USA (yau@uic.edu). Research partially supported by research grants from US Army Research Office and NSF.
} 
It can be shown that any Heisenberg manifold has constant step 2, i.e., $X_{1}, \ldots, X_{m}$ and their first brackets generate the tangent bundle $T \mathcal{M}$. The first concrete example of Heisenberg manifold is the Heisenberg group which was studied by Gaveau [12] and Strichartz [20]. A rather complete description of the geometry of Heisenberg group can be found in Beals, Gaveau, Greiner [2] and Calin, Chang, Greiner [8]. Moreover, if $(\mathcal{M}, \mathcal{D}, h)$ is a Heisenberg manifold then $\operatorname{dim} \mathcal{M}=n$ must be odd and $m$ must be even. The 2 -form $\Omega=d \omega$ is called the curvature 2 -form. It is known that if the distribution $\mathcal{D}$ is non-integrable then $\Omega \neq 0$.

The subRiemannian geometry deals with the geometry induced by the second order operator $\Delta_{X}=\sum_{j=1}^{m} X_{j}^{2}$. When $m=n, \Delta_{X}$ is the usual Laplace-Beltrami operator. It is well known that the Newtonian potential is

$$
N\left(\mathbf{x}, \mathbf{x}_{0}\right)=\frac{1}{(2-n)\left|\sigma_{n}\left(\mathbf{x}_{0}\right)\right| d^{n-2}\left(\mathbf{x}, \mathbf{x}_{0}\right)}, \quad n>2
$$

where $\left|\sigma_{n}\left(\mathbf{x}_{0}\right)\right|$ is the surface measure of the induced ball centered at $\mathbf{x}_{0}$ and $d\left(\mathbf{x}, \mathbf{x}_{0}\right)$ is the Riemannian distance between $\mathbf{x}$ and $\mathbf{x}_{0}$. It is known that $d\left(\mathbf{x}, \mathbf{x}_{0}\right) \approx\left|\mathbf{x}-\mathbf{x}_{0}\right|$ when $\mathbf{x}$ and $\mathbf{x}_{0}$ are sufficiently closed where $\left|\mathbf{x}-\mathbf{x}_{0}\right|$ is the Euclidean distance between $\mathbf{x}$ and $\mathbf{x}_{0}$. When $m<n$, the operator $\Delta_{X}$ is not elliptic. However, if $X_{1}, \ldots, X_{m}$ and a finite number of their Lie brackets generate $T \mathcal{M}$, then by a theorem of Hörmander [16] we know that $\Delta_{X}$ is hypoelliptic. In fact, Chow's theorem [10] ensures that every 2 points of $\mathcal{M}$ may be connected by a piecewise $C^{1}$ horizontal curve. This yields a distance and therefore a geometry which we shall call subRiemannian geometry. To see how remarkable Chow's theorem is, note that given two vector fields $X_{1}=\partial_{x_{1}}$ and $X_{2}=\partial_{x_{2}}$ in $\mathbb{R}^{3}$, there is no horizontal curve joining any two points $\left(x_{1}, x_{2}, t\right)$ and $\left(y_{1}, y_{2}, u\right)$ with $t \neq u$. For more general discussion of subRiemannian geometry, readers may consult the papers [6], [ 7], [10], [13] and [15]. Let $\mathcal{D}=\operatorname{span}\left\{X_{1}, X_{2}\right\}$ with $X_{1}=\partial_{x_{1}}+A_{1}(x) \partial_{t}$ and $X_{2}=\partial_{x_{2}}-A_{2}(x) \partial_{t}$ be two vector fields in $\mathbb{R}^{3}$. Here $A_{1}, A_{2}$ are two smooth functions defined on $\mathcal{M}=\mathbb{R}^{3}$ such that $\varphi(x):=\frac{\partial A_{1}}{\partial x_{2}}+\frac{\partial A_{2}}{\partial x_{1}} \neq 0$. Hence $(\mathcal{M}, \mathcal{D}, h)$ is a Heisenberg manifold since $\omega\left(\left[X_{1}, X_{2}\right]\right)=-\varphi(x)$ with $\omega=d t-A_{1}(x) d x_{1}+$ $A_{2}(x) d x_{2}$. In this and in a subsequent article, we shall study the following question:

"How many geodesics induced by $X_{1}$ and $X_{2}$ join two given points of $\mathcal{M}$ "?

By a geodesic we mean the projection of a bicharacteristic on the base. Bicharacteristics $\left(x_{1}(s), x_{2}(s), t(s), \xi_{1}(s), \xi_{2}(s), \theta(s)\right)$ are solutions of Hamilton's differential equations (see e.g., [2], [8] and [13]). The paper is written in two parts. In the first part, we first derive the Euler-Lagrange system which are induced by the operator $\Delta_{X}$ in section 2. Then we obtain solutions of the system in section 3 . We especially study the periodic solutions of the Euler-Lagrange system when $\varphi(x)$ is linear and the Lagrange multiplier formula in sections 4 and 5.

In the second part of the article, we investigate the difference between normal and abnormal geodesics. As we mention before, according to Chow's theorem that for every two points $P$ and $Q \in \mathcal{M}$ there is a piecewise $\mathcal{C}^{1}$ horizontal curve $\gamma:[0,1] \rightarrow \mathcal{M}$ such that $\gamma(0)=P$ and $\gamma(1)=Q$. Denote $\Gamma(P, Q)$ the set of such curves. For $\gamma \in \Gamma$, we let

$$
E(\gamma)=\frac{1}{2} \int_{0}^{1}\left[\dot{x}^{2}(s)+\dot{y}^{2}(s)\right] d s
$$

denote its energy. We are interested in the following variational problem: "find a curve $\gamma(s)$ of minimal energy in $\Gamma(P, Q)$ ".

In other words we are looking for a horizontal curve $\gamma(s)$ such that $\gamma(0)=P, \gamma(1)=Q$ 
and $E(\gamma)=\min \{E(\tilde{\gamma}): \tilde{\gamma} \in \Gamma(P, Q)\}$. This is a control problem. In sections 6 and 7 , we find conditions on a horizontal curve which satisfies Euler-Lagrange system but not the Hamilton's system. However, the solution indeed minimize the energy (and hence the arc-length) among all curves $\gamma \in \Gamma(P, Q)$, for points $P$ and $Q$ close enough.

2. A general 2-vector case. Let $A_{1}, A_{2} \in \mathcal{C}^{1}\left(\mathbb{R}^{3}\right)$ such that

$$
\varphi(x):=\frac{\partial A_{1}}{\partial x_{2}}+\frac{\partial A_{2}}{\partial x_{1}} \neq 0 .
$$

Consider $\mathcal{D}=\operatorname{span}\left\{X_{1}, X_{2}\right\}$, with

$$
X_{1}=\partial_{x_{1}}+A_{1}(x) \partial_{t}, \quad X_{2}=\partial_{x_{2}}-A_{2}(x) \partial_{t} .
$$

Since $\left[X_{1}, X_{2}\right]=-\varphi(x) \partial_{t} \notin \mathcal{D}$, the distribution $\mathcal{D}$ is not involutive and hence nonintegrable. The 1-connection form is

$$
\omega=d t-A_{1}(x) d x_{1}+A_{2}(x) d x_{2} .
$$

Since $\omega\left(\left[X_{1}, X_{2}\right]\right)=-\varphi(x) \neq 0$, the non-vanishing condition holds. Hence the vector fields (2.2) define a Heisenberg manifold on $\mathbb{R}^{3}$.

Let $\gamma=\left(x_{1}, x_{2}, t\right)$ be a curve in $\mathbb{R}^{3}$. Since

$$
\begin{aligned}
\dot{\gamma}= & \dot{x}_{1} \partial_{x_{1}}+\dot{x}_{2} \partial_{x_{2}}+\dot{t} \partial_{t} \\
= & \dot{x}_{1}\left(\partial_{x_{1}}+A_{1}(x) \partial_{t}\right)+\dot{x}_{2}\left(\partial_{x_{2}}-A_{2}(x) \partial_{t}\right) \\
& +\left(\dot{t}-A_{1}(x) \dot{x}_{1}+A_{2}(x) \dot{x}_{2}\right) \partial_{t} \\
= & \dot{x}_{1} X_{1}+\dot{x}_{2} X_{2}+\omega(\dot{\gamma}) .
\end{aligned}
$$

If $\gamma$ is a horizontal curve then $\dot{\gamma}=\dot{x}_{1} X_{1}+\dot{x}_{2} X_{2}$. The subRiemannian metric $g$ is chosen such that the vector fields $X_{1}$ and $X_{2}$ are orthonormal, so that the energy of the horizontal curve $c:[0, \tau] \rightarrow \mathbb{R}^{3}$ will be

$$
E(c)=\int_{0}^{\tau} \frac{1}{2} g(\dot{c}(s), \dot{c}(s)) d s=\frac{1}{2} \int_{0}^{\tau}\left(\dot{x}_{1}^{2}(s)+\dot{x}_{2}(s)^{2}\right) d s .
$$

Since we assume the curve horizontal, i.e., the curve satisfies the non-holonomic constraint $\omega(\dot{c})=0$, standard techniques regarding Lagrange multipliers yields the variational problem with the Lagrangian

$$
\begin{aligned}
L(x, t, \dot{x}, \dot{t}) & =\frac{1}{2}\left(\dot{x}_{1}^{2}+\dot{x}_{2}^{2}\right)+\lambda(s) \omega(\dot{c}) \\
& =\frac{1}{2}\left(\dot{x}_{1}^{2}+\dot{x}_{2}^{2}\right)+\lambda(s)\left(\dot{t}(s)-A_{1}(x) \dot{x}_{1}+A_{2}(x) \dot{x}_{2}\right) .
\end{aligned}
$$

For the general case of several Lagrange multipliers, see theorems 6.1 and 6.5. We are interested in the curves which are minimizers for the integral action

$$
c \rightarrow \int_{0}^{\tau} L(x, t, \dot{x}, \dot{t}) d s .
$$

These curves will be called normal geodesics. Their equations can be obtained from the Euler-Lagrange system of equations. Since

$$
\dot{\lambda}=\frac{d}{d s} \frac{\partial L}{\partial \dot{t}}=\frac{\partial L}{\partial t}=0
$$


it follows that the Lagrange multiplier $\lambda$ is constant along the solutions. The other two Euler-Lagrange equations

$$
\frac{d}{d s} \frac{\partial L}{\partial \dot{x}_{i}}=\frac{\partial L}{\partial x_{i}}, \quad i=1,2
$$

lead to the following ODE system:

$$
\begin{aligned}
& \ddot{x}_{1}(s)=\lambda \varphi(x) \dot{x}_{2}(s) \\
& \ddot{x}_{2}(s)=-\lambda \varphi(x) \dot{x}_{1}(s),
\end{aligned}
$$

where

$$
\varphi(x)=\frac{\partial A_{1}}{\partial x_{2}}+\frac{\partial A_{2}}{\partial x_{1}} .
$$

The $t$-component can be found by integrating in the horizontal constraint

$$
\dot{t}(s)=A_{1}(x) \dot{x}_{1}(s)-A_{2}(x) \dot{x}_{2}(s) .
$$

For general functions $A_{i}(x)$ an explicit solution for the system (2.4)-(2.5) is hard to find, unless the coefficient $\varphi(x)$ is of particular type. The case when $\varphi(x)$ has rotational symmetry or it is a constant can be found in [6]. Here we consider a different case where $\varphi(x)$ is linear.

3. Solving the Euler-Lagrange system with $\varphi(x)$ linear. In this section we shall solve the Euler-Lagrange system (2.4-2.5) explicitly in the case when $\varphi(x)=$ $a x_{1}+b x_{2}+c$ is a linear function with $a, b, c$ real constants. We shall show that the system can be reduced to the well-known pendulum equation

$$
\ddot{\theta}=-\omega^{2} \sin \theta
$$

where $\theta$ is the angle between the pendulum string and the downward vertical. The constant $\omega^{2}$ is equal to the quotient between the gravitational acceleration and the length of the pendulum string. The solution of the equation (3.7) satisfies the following expressions involving elliptic functions.

$$
\begin{aligned}
& \sin \frac{\theta}{2}=k \operatorname{sn}(\omega s, k) \\
& \cos \frac{\theta}{2}=\operatorname{dn}(\omega, k),
\end{aligned}
$$

where $k=\sin \frac{\alpha}{2}$ is the modulus of the elliptic functions. The positive constant $\alpha$ denotes the amplitude of the swing, i.e., $-\alpha \leq \theta(s) \leq \alpha$.

The qualitative features of the solution of the pendulum equation (3.7) are illustrated in the Figure 1. The reader may find more about this equation on p.35 in Calin and Chang [10].

The case when $\varphi(x)$ is linear covers a great deal of distributions which are spanned by the vector fields

$$
X_{1}=\partial_{x_{1}}+A_{1}(x) \partial_{t}, \quad X_{2}=\partial_{x_{2}}-A_{2}(x) \partial_{t},
$$




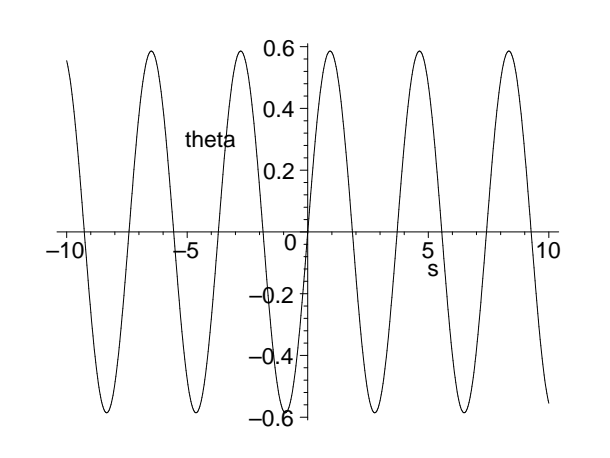

FIG. 1. The solution of $\ddot{\theta}=-3 \sin \theta$, with initial conditions $\theta(0)=0, \dot{\theta}(0)=1$.

with

$$
\frac{\partial A_{1}}{\partial x_{2}}+\frac{\partial A_{2}}{\partial x_{1}}=a x_{1}+b x_{2}+c .
$$

In particular, when $c=0$, this covers two important families of vector fields

$$
\begin{aligned}
& X_{1}=\partial_{x_{1}}+\left(a x_{1} x_{2}+f\left(x_{1}\right)\right) \partial_{t}, \\
& X_{2}=\partial_{x_{2}}-\left(b x_{1} x_{2}+g\left(x_{2}\right)\right) \partial_{t}
\end{aligned}
$$

and

$$
\begin{aligned}
& X_{1}=\partial_{x_{1}}+\left(\frac{1}{2} b x_{2}^{2}+f\left(x_{1}\right)\right) \partial_{t}, \\
& X_{2}=\partial_{x_{2}}-\left(\frac{1}{2} a x_{1}^{2}+g\left(x_{2}\right)\right) \partial_{t},
\end{aligned}
$$

with $f, g$ arbitrary smooth real-valued functions. We note that the second pair of vector fields have quadratic coefficients in $x_{1}$ and $x_{2}$, which makes the model more interesting.

When $a=b=0$ and $c \neq 0$, choosing $A_{1}=\frac{c}{2} x_{2}$ and $A_{2}=\frac{c}{2} x_{1}$ leads to the Heisenberg group case. In this case, the Euler-Lagrange system becomes

$$
\ddot{x}_{1}=c \lambda \dot{x}_{2}, \quad \ddot{x}_{2}=-c \lambda \dot{x}_{1}, \quad \dot{t}=\frac{c}{2}\left(x_{2} \dot{x}_{1}-x_{1} \dot{x}_{2}\right) .
$$

All the above cases can be solved using the following method of conservation of energy. The kinetic energy $E=\frac{1}{2}\left(\dot{x}_{1}^{2}+\dot{x}_{2}^{2}\right)$ is a first integral of motion for the system (2.4-2.5). This follows if we multiply the first equation of the system by $\dot{x}_{1}$ and the second equation by $\dot{x}_{2}$ and add

$$
\underbrace{\dot{x}_{1} \ddot{x}_{1}+\dot{x}_{2} \ddot{x}_{2}}_{\frac{d}{d s}\left(\frac{\dot{x}_{1}^{2}+\dot{x}_{2}^{2}}{2}\right)}=\lambda \varphi(x) \dot{x}_{1} \dot{x}_{2}-\lambda \varphi(x) \dot{x}_{1} \dot{x}_{2}=0 .
$$


Let $s$ denote the arc length parameter. Then $E=R^{2} / 2$ and $\dot{x}_{1}(s)^{2}+\dot{x}_{2}(s)^{2}=R^{2}$ with $R>0$ constant. It follows that $\left(\dot{x}_{1}, \dot{x}_{2}\right)$ belongs to the circle centered at the origin with radius $R$ and hence there is an argument function $\psi$ such that

$$
\dot{x}_{1}(s)=R \cos \psi(s), \quad \dot{x}_{2}=R \sin \psi(s) .
$$

Substituting back in the system (2.4-2.5) yields

$$
\begin{aligned}
-R \sin \psi \dot{\psi} & =\lambda \varphi(x) R \sin \psi \\
R \cos \psi \dot{\psi} & =-\lambda \varphi(x) R \cos \psi .
\end{aligned}
$$

Multiplying the first equation by $\sin \psi$ and the second by $\cos \psi$ we obtain

$$
\dot{\psi}=-\lambda \varphi(x) \text {. }
$$

Until this moment we haven't use the particular form of $\varphi(x)$ yet. Hence the equation (3.11) works in the general case, but cannot be solved in all cases. In the case when $\varphi(x)=a x_{1}+b x_{2}+c\left(a^{2}+b^{2} \neq 0\right)$, additional differentiation of equation (3.11) together with the use of equations (3.10) leads to an ODE in $\psi$ :

$$
\begin{aligned}
\ddot{\psi} & =-\lambda\left(a \dot{x}_{1}(s)+b \dot{x}_{2}(s)\right) \\
& =-\lambda(a \cos \psi(s)+b \sin \psi(s)) \\
& =-\lambda \sqrt{a^{2}+b^{2}}\left(\sin \phi_{0} \cos \psi+\cos \phi_{0} \sin \psi\right) \\
& =-\lambda \sqrt{a^{2}+b^{2}} \sin \left(\psi+\phi_{0}\right),
\end{aligned}
$$

where $\phi_{0}=\tan ^{-1}\left(\frac{a}{b}\right)$. Let $\omega^{2}:=\lambda \sqrt{a^{2}+b^{2}}$ and set $\theta(s)=\psi(s)+\phi_{0}$. Then the equation (3.12) becomes the pendulum equation (3.7). The solution of the system (2.4-2.5) can be obtained by integration in terms of the pendulum function:

$$
\begin{aligned}
& x_{1}(s)=x_{1}(0)+\int_{0}^{s} \cos \left(\theta(u)-\phi_{0}\right) d u \\
& x_{2}(s)=x_{2}(0)+\int_{0}^{s} \sin \left(\theta(u)-\phi_{0}\right) d u .
\end{aligned}
$$

The $t$-component can also be obtained by integration in the equation (2.6):

$$
t(s)=t(0)+\int_{0}^{s}\left[A_{1}(u) \cos \left(\theta(u)-\phi_{0}\right)-A_{2}(u) \sin \left(\theta(u)-\phi_{0}\right)\right] d u .
$$

Proposition 3.1. Let $\varphi(x)=a x_{1}+b x_{2}+c$. Then the solution of the system (2.4-2.5) is

$$
\begin{aligned}
& x_{1}(s)=x_{1}(0)-\frac{1}{\sqrt{a^{2}+b^{2}}}\left(a s-\frac{2}{\omega}(a E(\omega s, k)+k b(\operatorname{cn}(\omega s, k)-1))\right), \\
& x_{2}(s)=x_{2}(0)+\frac{1}{\sqrt{a^{2}+b^{2}}}\left(b s-\frac{2}{\omega}(b E(\omega s, k)+k a(c n(\omega s, k)-1))\right),
\end{aligned}
$$


where $E(u, k)=\int_{0}^{u} d n^{2}(s) d u$ is the Jacobi's epsilon function, $\omega^{2}=|\lambda| \sqrt{a^{2}+b^{2}}$ and $k=\sin \frac{\alpha}{2}$, with $\alpha=\max \theta(s)$.

Proof. We shall integrate in (3.13)-(3.14) using the following formulas (see Lawden [17], p.36, p.62)

$$
\begin{aligned}
\int \operatorname{dn}^{2} u d u & =u-k^{2} \int \operatorname{sn}^{2} u d u, \\
\frac{d}{d u} \operatorname{cn} u & =-\operatorname{sn} u \operatorname{dn} u .
\end{aligned}
$$

Using (3.8)-(3.9) yields

$$
\begin{aligned}
\cos \left(\theta-\phi_{0}\right) & =\cos \theta \cos \phi_{0}+\sin \theta \sin \phi_{0} \\
& =\left(1-2 \sin ^{2} \frac{\theta}{2}\right) \cos \phi_{0}+2 \sin \frac{\theta}{2} \cos \frac{\theta}{2} \sin \phi_{0} \\
& =\left(1-2 k^{2} \operatorname{sn}^{2}(\omega s, k)\right) \cos \phi_{0}+2 k \operatorname{sn}(\omega s, k) \operatorname{dn}(\omega s, k) \sin \phi_{0} .
\end{aligned}
$$

Integrating yields

$$
\begin{aligned}
& \int_{0}^{s} \cos \left(\theta(u)-\phi_{0}\right) d u \\
= & \left(\cos \phi_{0}\right) s-2 k^{2} \cos \phi_{0} \int_{0}^{s} \operatorname{sn}^{2}(\omega u, k) d u+2 k \sin \phi_{0} \int_{0}^{s} \operatorname{sn}(\omega u, k) \operatorname{dn}(\omega u, k) d u \\
= & \left(\cos \phi_{0}\right) s-\frac{2}{\omega} k^{2} \cos \phi_{0} \int_{0}^{\omega s} \operatorname{sn}^{2}(v, k) d v+\frac{2}{\omega} k \sin \phi_{0} \int_{0}^{\omega s} \operatorname{sn}(v, k) \operatorname{dn}(v, k) d v \\
= & \left(\cos \phi_{0}\right) s+\frac{2}{\omega} \cos \phi_{0}(\omega s-E(\omega s, k))-\frac{2 k}{\omega} \sin \phi_{0}(\operatorname{cn}(\omega s, k)-1) \\
= & -\left(\cos \phi_{0}\right) s+\frac{2}{\omega} \cos \phi_{0} E(\omega s, k)-\frac{2 k}{\omega} \sin \phi_{0}(\operatorname{cn}(\omega s)-1) \\
= & -\frac{1}{\sqrt{a^{2}+b^{2}}}\left(a s-\frac{2}{\omega}(a E(\omega s, k)+k b(\operatorname{cn}(\omega s, k)-1))\right),
\end{aligned}
$$

which leads to the expression of $x_{1}(s)$ given in the conclusion. In order to obtain the expression for $x_{2}(s)$ we compute

$$
\begin{aligned}
\sin \left(\theta-\phi_{0}\right) & =\sin \theta \cos \phi_{0}-\cos \theta \sin \phi_{0} \\
& =2 \sin \frac{\theta}{2} \cos \frac{\theta}{2} \cos \phi_{0}-\left(1-2 \sin ^{2} \frac{\theta}{2}\right) \sin \phi_{0} \\
& =2 k \operatorname{sn}(\omega s, k) \operatorname{dn}(\omega s, k) \cos \phi_{0}-\sin \phi_{0}+2 k^{2} \sin \phi_{0} \operatorname{sn}^{2}(\omega s, k) .
\end{aligned}
$$


Integrating yields

$$
\begin{aligned}
& \int_{0}^{s} \sin \left(\theta(u)-\phi_{0}\right) d u \\
= & 2 k \cos \phi_{0} \int_{0}^{s} \operatorname{sn}(\omega u, k) \operatorname{dn}(\omega u, k) d u-s \sin \phi_{0}+2 k^{2} \sin \phi_{0} \int_{0}^{s} \operatorname{sn}^{2}(\omega u, k) d u \\
= & \frac{2 k}{\omega} \cos \phi_{0} \int_{0}^{\omega s} \operatorname{sn}(v, k) \operatorname{dn}(v, k) d v-s \sin \phi_{0}+\frac{2}{\omega} \sin \phi_{0} k^{2} \int_{0}^{\omega s} \operatorname{sn}^{2}(\omega s, k) d v \\
= & \frac{2 k}{\omega} \cos \phi_{0}(1-\operatorname{cn}(\omega s, k))+s \sin \phi_{0}-\frac{2}{\omega} \sin \phi_{0} E(\omega s, k) \\
= & \frac{1}{\sqrt{a^{2}+b^{2}}}\left(\frac{2 k}{\omega} a(1-\operatorname{cn}(\omega s, k))+b s-\frac{2}{\omega} b E(\omega s, k)\right) \\
= & \frac{1}{\sqrt{a^{2}+b^{2}}}\left(b s-\frac{2}{\omega}(b E(\omega s, k)+k a(\operatorname{cn}(\omega s, k)-1))\right),
\end{aligned}
$$

which leads to the expression of $x_{2}(s)$.

REMARK 3.2. For the Heisenberg group case, one has

$$
\dot{x}_{1}(s)=R \cos \psi(s), \quad \dot{x}_{2}(s)=R \sin \psi(s) .
$$

Then

$$
\ddot{x}_{1}(s)=-R \sin \psi(s) \dot{\psi}(s), \quad \ddot{x}_{2}(s)=R \cos \psi(s) \dot{\psi}(s),
$$

and the Euler-Lagrange system becomes

$$
\sin \psi(s)(c \lambda+\dot{\psi})=0, \quad \cos \psi(s)(c \lambda+\dot{\psi})=0 .
$$

Adding the squares, yields $\dot{\psi}(s)=-c \lambda$. Integrating, we obtain

$$
\psi(s)=-c \lambda s-\psi(0)=-c \lambda s-\psi_{0} .
$$

Hence one has

$$
x_{1}(s)=\frac{R}{c \lambda} \sin \left(c \lambda s+\psi_{0}\right)+x_{1}(0), \quad x_{2}(s)=\frac{R}{c \lambda} \cos \left(c \lambda s+\psi_{0}\right)+x_{2}(0) .
$$

This is a parametric equation of a circle centered at $\left(x_{1}(0), x_{2}(0)\right)$ with radius $\frac{R}{c \lambda}$. This tells us the projection of the solution of the Euler-Lagrange system is periodic.

\section{Periodic solutions in the case $\varphi(x)$ linear.}

- The geometric interpretation of $\psi$. From the Physics point of view $\theta$ measures the angle between the pendulum string and the vertical direction and it is a periodic function.

Dividing the equations (3.10) yields

$$
\tan \psi(s)=\frac{\dot{x}_{2}}{\dot{x}_{1}}=\frac{d x_{2}}{d x_{1}},
$$


i.e., $\psi(s)$ is the angle between the tangent line to the graph of $x_{2}=x_{2}\left(x_{1}\right)$ at the point $\left(x_{1}(s), x_{2}(s)\right)$. Since $\theta(s)$ is periodic, using that $\theta(s)=\psi(s)+\phi_{0}$, it follows that the angle $\psi(s)$ will be also periodic. We state this in the following:

Proposition 4.1. There is a positive constant $T>0$ such that $\psi(s+T)=\psi(s)$, for all $s>0$.

The following result will be useful:

Lemma 4.2. Let $f$ be a smooth real function with a periodic derivative, i.e., $f^{\prime}(x)=f^{\prime}(x+T)$, for all $x \in \mathbb{R}$, with $T>0$. Then

$$
f(x+m T)=f(x)+m(f(T)-f(0)), \quad \forall m \in \mathbb{Z} .
$$

In particular, if $f(0)=f(T)$, then the function $f$ is periodic with the period $T$.

Proof. Let $g(x)=f^{\prime}(x)$ be the derivative function. From the Fundamental Theorem of Calculus we have

$$
f(x)=f(0)+\int_{0}^{x} g(u) d u .
$$

Replacing $x$ by $x+m T$ and using that $g$ is periodic yields

$$
\begin{aligned}
f(x+m T) & =f(0)+\int_{0}^{x+m T} g(u) d u \\
& =f(0)+\int_{0}^{x} g(u) d u+\int_{x}^{x+m T} g(u) d u \\
& =f(x)+\int_{x}^{x+m T} g(u) d u \\
& =f(x)+\int_{0}^{m T} g(u) d u \\
& =f(x)+m \int_{0}^{T} g(u) d u \\
& =f(0)+m(f(T)-f(0)) .
\end{aligned}
$$

The second part is obvious.

Proposition 4.3. (i) If $x_{1}(0)=x_{1}(T)$ and $x_{2}(0)=x_{2}(T)$ then the functions $x_{i}$ are periodic with period $T$ :

$$
x_{1}(s+m T)=x_{1}(s), \quad x_{2}(s+m T)=x_{2}(s), \quad \forall s \geq 0, m \in \mathbb{Z} .
$$

This corresponds to a closed solution in the $x$-plane, see Figure 2.

(ii) If $x_{1}(0) \neq x_{1}(T), x_{2}(0) \neq x_{2}(T)$, we have

$$
\begin{aligned}
& x_{1}(s+m T)=x_{1}(s)+m\left(x_{1}(T)-x_{1}(0)\right), \\
& x_{2}(s+m T)=x_{2}(s)+m\left(x_{2}(T)-x_{2}(0)\right) .
\end{aligned}
$$

In particular, the solution is unbounded and not closed, see Figure 3.

Proof. Since $\psi(s)$ is periodic with period $T$, then $x_{1}(s)=\cos (\psi(s))$ and $x_{2}(s)=$ $\cos (\psi(s))$ will be periodic with period $T$. Applying Lemma 4.2 we obtain the desired results. 


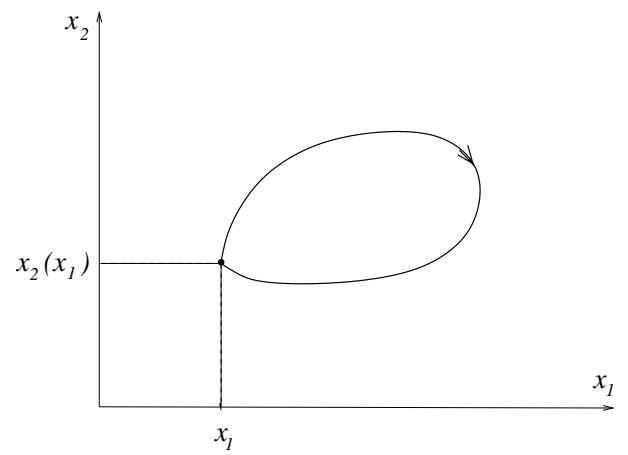

$x_{1}(0)=x_{1}(T)$

FiG. 2. Closed solution.

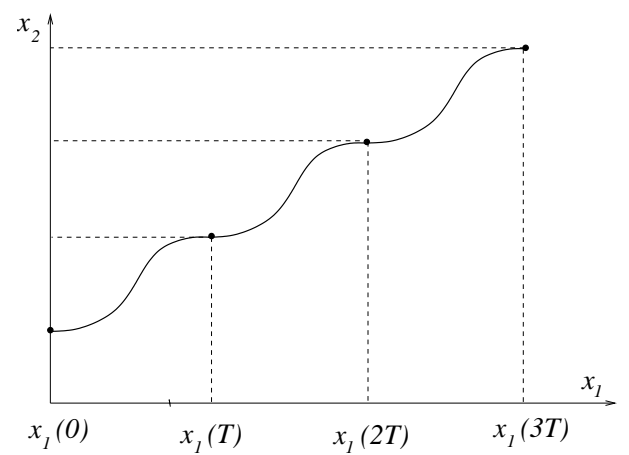

$x_{1}(T)>x_{1}(0)$

FIG. 3. Unbounded solution.

- Periodic solutions. Now we shall assume that $\varphi(x)=a x_{1}+b x_{2}+c$, with $a, b, c \in \mathbb{R}$. Using Propositions 4.3 and 3.1 we shall characterize the periodic solutions $x(s)=\left(x_{1}(s), x_{2}(s)\right)$. In order to have periodic solutions we need to find $T>0$ such that

$$
x_{1}(T)=x_{1}(0), \quad x_{2}(T)=x_{2}(0) .
$$

Using Proposition 3.1, the period $T$ must satisfy both equations

$$
\begin{aligned}
& a T-\frac{2}{\omega}(a E(\omega T, k)+k b(\operatorname{cn}(\omega T, k)-1))=0 \\
& b T-\frac{2}{\omega}(b E(\omega T, k)+k a(\operatorname{cn}(\omega T, k)-1))=0 .
\end{aligned}
$$

The solution $\theta(s)$ of the pendulum equation $\ddot{\theta}=-\omega^{2} \sin \theta$ satisfies

$$
\sin \frac{\theta}{2}=k \operatorname{sn}(\omega s, k)
$$

where $k=\sin \frac{\alpha}{2}$. This means that $\sin \frac{\theta}{2}$ oscillates with amplitude $\sin \frac{\alpha}{2}$ and period

$$
T=\frac{4 K}{\omega} \quad \Leftrightarrow \quad \omega T=4 K,
$$

where

$$
K=K(k)=\int_{0}^{\pi / 2} \frac{d u}{\sqrt{1-k^{2} \sin ^{2} u}}
$$

is a complete elliptic integral. Using $c n(4 K, k)=1$ and the addition property for the Jacobi's epsilon function

$$
E(2 m K)=2 m E,
$$


where $E=E(k)=\int_{0}^{K} \operatorname{dn}^{2} u d u$, both equations (4.16)-(4.17) can be reduced to the equation

$$
2 E(k)-K(k)=0,
$$

where we assumed $a, b \neq 0$. Since the function

$$
[0,1) \ni k \rightarrow K(k)
$$

is increasing with $K(0)=\pi / 2, K(1,-)=+\infty$ (Figure 4), and the function

$$
[0,1] \ni k \rightarrow E(k)
$$

is decreasing, with $E(0)=\pi / 2$ and $E(1)=1$ (Figure 5), it follows that the function

$$
[0,1) \ni k \rightarrow g(k)=2 E(k)-K(k)
$$

is decreasing, having a vertical asymptote at $k=1$ (Figure 6).

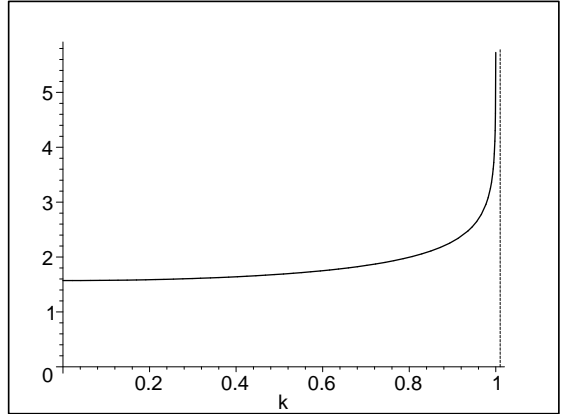

FIG. 4. The graph of $K(k)$.

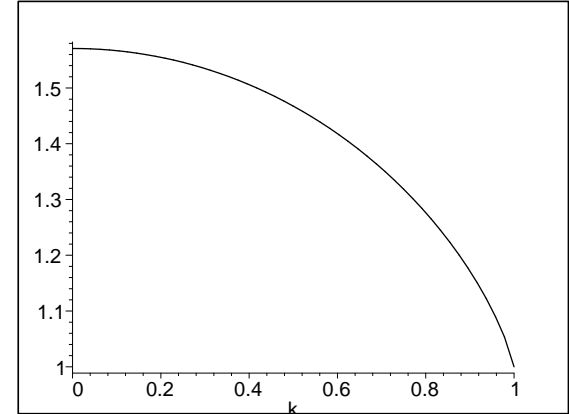

FIG. 5. The graph of $E(k)$.

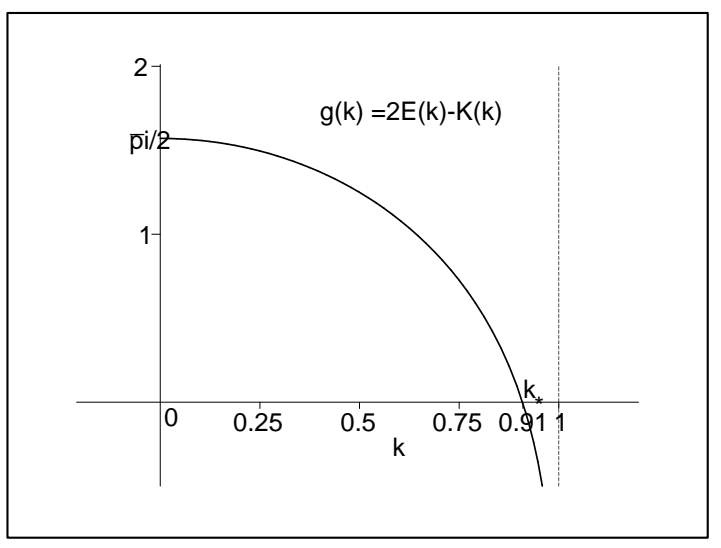

FIG. 6. The solution of $2 E(k)-K(k)=0$.

Since $g(0)=\pi / 2$ and $g(1,-)=-\infty$, it follows that the equation (4.18), i.e., $g(k)=0$ has a unique solution $k=k^{*} \approx 0.91$, and using $\sin \frac{\alpha}{2}=k^{*}$ yields the 
amplitude $\alpha^{*}=2 \arcsin k^{*} \approx 2.28$. Proposition 3.1 provides now the equations for the periodic solutions

$$
\begin{aligned}
& x_{1}(s)=x_{1}(0)-\frac{1}{\sqrt{a^{2}+b^{2}}}\left(a s-\frac{2}{\omega}\left(a E\left(\omega s, k^{*}\right)+k^{*} b\left(\operatorname{cn}\left(\omega s, k^{*}\right)-1\right)\right)\right) \\
& x_{2}(s)=x_{2}(0)+\frac{1}{\sqrt{a^{2}+b^{2}}}\left(b s-\frac{2}{\omega}\left(b E\left(\omega s, k^{*}\right)+k^{*} a\left(\operatorname{cn}\left(\omega s, k^{*}\right)-1\right)\right)\right) .
\end{aligned}
$$

It is interesting that the period $T=4 K\left(k^{*}\right) / \omega$, where $K\left(k^{*}\right) \approx 2.32$, does not depend on the initial point $x(0)$.

In the next section we shall relate the Lagrange multiplier $\lambda$ to the length of the periodic solutions.

5. The Lagrange multiplier formula. We shall consider the case of general periodic solutions. In this case an important feature is the total curvature of the unit speed curve $x(s)=\left(x_{1}(s), x_{2}(s)\right):[0, L] \rightarrow \mathbb{R}^{2}$ defined by

$$
\kappa_{T}=\int_{0}^{L} \kappa(s) d s
$$

where $\kappa(s)$ denotes the plane curvature of the plane curve $x(s) .{ }^{1}$ If $x(s)$ is a plane, closed curve, by Fenchel's formula (see Millman and Parker [18]) we have

$$
\kappa_{T}=2 m \pi, \quad m=1,2, \ldots
$$

where $m$ is the rotation index of the curve (see Figure 7).

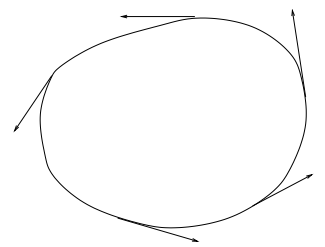

(a)

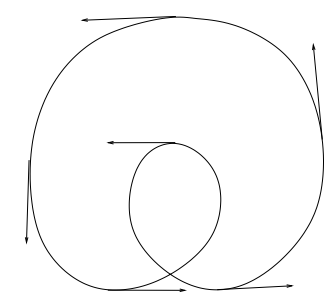

(b)

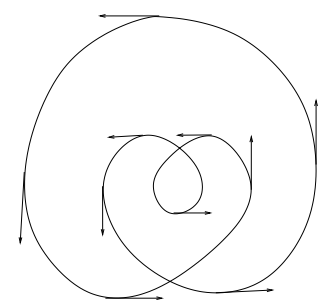

(c)

FIG. 7. Plane closed curves with index $m$ : (a) $m=1$, (b) $m=2$, (c) $m=3$.

By Euler's formula, the curvature of a plane unit-speed curve $x(s)$ is given by

$$
\kappa(s)=\frac{d \psi}{d s},
$$

where $\psi(s)$ is the angle made by the velocity vector field along the curve with the horizontal direction.

\footnotetext{
${ }^{1}$ The curvature of the plane unit speed curve $x(s)$ is defined by $\kappa(s)=\left|T^{\prime}(s)\right|$, where $T(s)=x^{\prime}(s)$.
} 


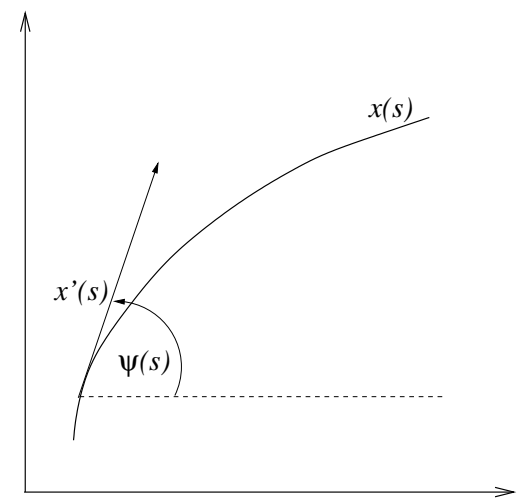

FIG. 8. The angle $\psi(s)$.

Using (5.20) and (3.11) yields the following formula for the plane curvature

$$
\kappa(s)=-\lambda \varphi(x(s))
$$

Integrating and using (5.19) we obtain

$$
2 m \pi=-\lambda_{m} \int_{0}^{L_{m}} \varphi(x(s)) d s=-\lambda_{m} \int_{0}^{L_{m}} \Omega_{12}(x(s)) d s
$$

where $\Omega_{12}=\Omega\left(X_{1}, X_{2}\right)=d \omega\left(\left[X_{1}, X_{2}\right]\right)$ and $L_{m}$ is the length of the solution which corresponds to the Lagrange multiplier $\lambda_{m}$.

Proposition 5.1. The Lagrange multiplier $\lambda$ has an extrinsic character, i.e., depends on the 1-connection form $\omega$ :

$$
\lambda_{m}=-\frac{1}{2 m \pi} \int_{0}^{L_{m}} d \omega\left(X_{1}, X_{2}\right)(x(s)) d x .
$$

EXAMPLE 5.1. In the three dimensional Heisenberg group case we have $d \omega\left(X_{1}, X_{2}\right)=\Omega_{12}=4$ and the above formula provides

$$
L_{m}=\frac{m \pi}{2}\left|\lambda_{m}\right|, \quad m=1,2,3, \ldots
$$

which is a formula for the length of a closed solution in terms of Lagrange multiplier. The smallest multipler $\lambda_{1}$ yields the Carnot-Caratheodory distance, see Calin, Chang and Greiner [5].

6. Abnormal minimizers. The subject of non-holonomic constraints in its Lagrangian formalism approach is the core of optimal control. Since we are dealing with curves (one parameter maps), only the one-dimensional Lagrangian problems will be of interest for us. For a more detailed approach of the subject the reader can consult the works of, e.g., Bliss [3], Bolza [4], Funk [11], and Sagan [19]. The theory of Lagrange's multiplier rule for minimizers of single integrals is quite complete and 
we shall follow M. Giaquinta and S. Hildebrandt [14] in our brief presentation. The reader can consult the proofs presented therein.

The variational problem consists in finding a mapping $u \in C^{2}\left([a, b], \mathbb{R}^{n}\right)$, which is a minimizer for the integral

$$
\mathcal{F}(u)=\int_{a}^{b} F(s, u(s), \dot{u}(s)) d s,
$$

under $r(r<n)$ functionally independent non-holonomic constraints

$$
G^{i}(s, u(s), \dot{u}(s))=0, \quad i=1, \ldots, r,
$$

i.e., constraints which satisfy the condition

$$
\operatorname{rank}\left(\frac{\partial G^{i}}{\partial \dot{u}^{j}}\right)_{i, j}=r .
$$

The existence of the Lagrange multipliers functions is stated in the following result (see p.117 in [14]).

Theorem 6.1 (Lagrange multiplier rule I). Let $u \in C^{2}\left([a, b], \mathbb{R}^{n}\right)$ be a minimizer of the above integral functional $\mathcal{F}(u)$ under the non-holonomic constraints (6.21) satisfying condition (6.22), and suppose that $F, G^{1}, \ldots, G^{r}$ are of class $C^{3}$. Then there exist a constant $\ell_{0}$ (which can be taken zero or one) and functions $\lambda_{1}(s), \ldots, \lambda_{r}(s) \in C^{1}([a, b])$ such that $u$ is an extremal of the unconstrained variational integral

$$
\mathcal{F}^{*}(u)=\int_{a}^{b} F^{*}(s, u, \dot{u}) d s,
$$

with the Lagrangian

$$
F^{*}(s, z, p)=\ell_{0} F(s, z, p)+\sum_{j=1}^{r} \lambda_{j}(s) G^{j}(s, z, p) .
$$

The case $\ell_{0}=1$ is called the principal case, and it is of particular importance. In this case the minimizer $u$ satisfies the Euler-Lagrange equations

$$
\frac{d}{d s}\left(\frac{\partial F^{*}}{\partial \dot{u}^{i}}\right)=\frac{\partial F^{*}}{\partial u^{i}}, \quad i=1, \ldots, n .
$$

In the exceptional case $\ell_{0}=0$ the Lagrangian $F$ does not appear in the EulerLagrange equations and we obtain the following equations

$$
\frac{d}{d s}\left(\sum_{j=1}^{r} \lambda_{j}(s) G_{\dot{u}_{i}}^{j}\right)=\sum_{j=1}^{r} \lambda_{j}(s) G_{u_{i}}^{j}, \quad i=1, \ldots, n .
$$

where

$$
G_{u_{i}}^{j}=\frac{\partial G^{j}}{\partial u_{i}}, \quad G_{\dot{u}_{i}}^{j}=\frac{\partial G^{j}}{\partial \dot{u}_{i}} .
$$

We have the following result (see p.117 in [14]). 
Lemma 6.2. If $\ell_{0}=0$, then there is a nontrivial solution $\lambda(s)=$ $\left(\lambda_{1}(s), \ldots, \lambda_{r}(s)\right)$ of $(6.23)$.

Motivated by the previous result Hahn introduced the following terminology (see p.118 of [14]).

Definition 6.3. A minimizer of $\mathcal{F}$ under the constraints $G^{j}(x, u, \dot{u})=0, j=$ $1, \ldots, r$, is said to be abnormal if there is a nontrivial $C^{1}$-solution $\lambda=\left(\lambda_{1}, \ldots, \lambda_{r}\right)$ of (6.23). Otherwise $u$ is called a normal minimizer.

Lemma 6.2 can be also stated as follows:

Proposition 6.4. If $\ell_{0}=0$, then $u$ is an abnormal minimizer. The abnormal minimizers are solutions of (6.23), which satisfy the non-holonomic constraints $G^{j}(s, u, \dot{u})=0, j=1, \ldots, r$.

The following result deals with the normal minimizers which was originally proved by Hahn and Bolza. The reference can be found in p.118 in [14].

THEOREM 6.5 (Lagrange multiplier rule II). If $u$ is a normal minimizer of $\mathcal{F}$ under the constraints $G^{j}(s, u, \dot{u})=0, j=1, \ldots, r$, then $u$ is an $F^{*}$-extremal for the Lagrangian

$$
F^{*}(s, z, p)=F(s, z, p)+\sum_{j=1}^{r} \lambda_{j}(s) G^{j}(s, z, p)
$$

and the multipliers $\lambda_{1}, \ldots, \lambda_{r}$ are uniquely determined by $u$.

In the case of subRiemannian geometry the minimizers $u$ are elements of $C^{2}\left([0, \tau], \mathbb{R}^{n}\right)$, while the non-holonomic constraints are given by $r$ one-forms $\theta_{j}$ as follows

$$
G^{j}(u, \dot{u})=\theta^{j}(\dot{u})
$$

If $\theta^{j}=\theta_{i}^{j} d x_{i}$, is a representation in local coordinates, then the condition (6.22) becomes $\operatorname{rank}\left(\theta_{j}^{i}\right)=r$, i.e., the one-forms are functionally independent.

The Lagrangian $F$ is the energy in the subRiemannian metric $h$ and it does not depend explicitly on $s$

$$
F(u, \dot{u})=\frac{1}{2} h(\dot{u}, \dot{u}),
$$

with horizontal velocity vector field $\dot{u}(s) \in \mathcal{D}_{u(s)}$. The normal minimizers are extremals for the Lagrangian

$$
F^{*}(u, \dot{u})=\frac{1}{2} h(\dot{u}, \dot{u})+\sum_{j=1}^{r} \lambda_{j} \theta^{j}(\dot{u}),
$$

while the abnormal minimizers are extremals for

$$
F^{*}(u, \dot{u})=\sum_{j=1}^{r} \lambda_{j} \theta^{j}(\dot{u})
$$

where $\lambda_{j} \in C^{1}([0, \tau])$ are Lagrange multipliers functions, not necessary constant. 
Proposition 6.6. In the case of the non-holonomic constraints $\theta^{j}(\dot{u})=0, j=$ $1, \ldots, r$, the equations of the abnormal minimizers are

$$
\sum_{j=1}^{r} \dot{\lambda}_{j}(s) \theta_{i}^{j}(u(s))=2 \sum_{j=1}^{r} \lambda_{j}(s) \Theta_{i r}^{j}(u(s)) \dot{u}^{r}, \quad i=1, \ldots, n,
$$

where $\Theta^{j}=d \theta^{j}$ and $\Theta_{i r}^{j}=\Theta^{j}\left(\partial_{i}, \partial_{r}\right)$.

Proof. Since the non-holonomic constraints are $G^{j}(u)=\theta^{j}(\dot{u})=\theta_{k}^{j} \dot{u}^{k}$ we have $G_{u_{i}}^{j}=\frac{\partial \theta_{k}^{j}}{\partial x_{i}} \dot{u}^{k}$ and $G_{\dot{u}_{i}}^{j}=\theta_{i}^{j}$ and the left side of equation (6.23) becomes

$$
\frac{d}{d s}\left(\sum_{j} \lambda_{j}(s) \theta_{i}^{j}(u(s))\right)=\sum_{j} \dot{\lambda}_{j}(s) \theta_{i}^{j}(u(s))+\sum_{j} \lambda_{j}(s) \frac{\partial \theta_{r}^{j}}{\partial x^{r}} \dot{u}^{r} .
$$

The right side of equation (6.23) can be written as

$$
\sum_{j, k} \lambda_{j}(s) G_{u_{i}}^{j}=\sum_{j, k} \lambda_{j}(s) \frac{\partial \theta_{r}^{j}}{\partial x^{i}} \dot{u}^{r} .
$$

Equating the expressions (6.25) and (6.26) and using that the components $\Theta_{i r}^{j}=\frac{\partial \theta_{r}^{j}}{\partial x^{r}}$ are skew-symmetric in the lower indices, yields equations (6.24).

COROLlaRY 6.7. (i) The abnormal minimizers are horizontal curves which satisfy the equation

$$
\dot{\lambda}(s) \theta_{i}(u(s))=2 \lambda(s) \Theta_{i r}(u(s)) \dot{u}^{r}(s), \quad i=1, \ldots, n,
$$

for some Lagrange multiplier function $\lambda(s)$.

(ii) If there is $\left(i_{0}, r_{0}\right)$ such that the component $\Theta_{i_{0} r_{0}} \equiv 0$, then the Lagrange multiplier function $\lambda$ is a constant.

(iii) If besides the conditions in (ii), we have $\operatorname{det} \Theta_{i j}(x) \neq 0$ for any $x$, then there are no abnormal minimizers.

Proof. (i) It follows from the above proposition in the case of only one nonholonomic constraint given by the one-form $\theta$, with the exterior derivative $\Theta=d \theta$.

(ii) Since the right hand side of (6.27) vanishes for $(i, r)=\left(i_{0}, r_{0}\right)$ and $\lambda$ does not depend on the pair of indices $(i, r)$, it follows that the left hand side of (6.27) vanishes too, so that $\dot{\lambda}(s)=0$, i.e., $\lambda$ is a constant.

(iii) If $\lambda$ is a non-zero constant, then $\Theta_{i r}(u) \dot{u}^{r}=0$ with the unique solution $\dot{u}(s)=0$, which means that $u(s)$ is a constant, contradiction. Hence $\lambda=0$ and there are no abnormal minimizers.

EXAMPLE 6.1. Let $\theta=2 x_{1} d x_{2}+2 x_{2} d x_{3}+2 x_{3} d x_{1}$. Then

$$
\Theta=d \theta=2 d x_{1} \wedge d x_{2}+2 d x_{2} \wedge d x_{3}+2 d x_{3} \wedge d x_{1},
$$

with the non-singular components matrix

$$
\Theta_{i j}=\left(\begin{array}{ccc}
0 & 1 & 1 \\
-1 & 0 & 1 \\
-1 & -1 & 0
\end{array}\right)
$$

Hence there are no abnormal minimizers subject to the non-holonomic constraint $\theta(\dot{u})=0$. 
7. Abnormal minimizers (continued). We shall investigate the abnormal minimizers in the case of the vector fields

$$
X_{1}=\partial_{x_{1}}+A_{1}(x) \partial_{t}, \quad X_{2}=\partial_{x_{2}}-A_{2}(x) \partial_{t} .
$$

The Lagrangian is

$$
L(x, t, \dot{x}, \dot{t})=\frac{\ell_{0}}{2}\left(\dot{x}_{1}^{2}+\dot{x}_{2}^{2}\right)+\lambda(s)\left(\dot{t}-A_{1}(x) \dot{x}_{1}+A_{2}(x) \dot{x}_{2}\right)
$$

and in order to obtain the abnormal minimizers we set $\ell_{0}=0$ and write the EulerLagrange equations for the Lagrangian

$$
L^{*}(x, t, \dot{x}, \dot{t})=\lambda(s)\left(\dot{t}-A_{1}(x) \dot{x}_{1}+A_{2}(x) \dot{x}_{2}\right) .
$$

From $\lambda(s)=\frac{\partial L^{*}}{\partial \dot{t}}$ and $\frac{\partial L^{*}}{\partial t}=0$ it follows that $\dot{\lambda}(s)=0$ i.e., the Lagrange multiplier $\lambda$ is constant. Using that

$$
\begin{gathered}
\frac{\partial L^{*}}{\partial \dot{x}_{1}}=-\lambda A_{1}, \quad \frac{\partial L^{*}}{\partial \dot{x}_{2}}=-\lambda A_{2}, \\
\frac{\partial L^{*}}{\partial x_{1}}=-\lambda \frac{\partial A_{1}}{\partial x_{1}} \dot{x}_{1}+\lambda \frac{\partial A_{2}}{\partial x_{1}} \dot{x}_{2}, \quad \frac{\partial L^{*}}{\partial x_{2}}=-\lambda \frac{\partial A_{1}}{\partial x_{2}} \dot{x}_{1}+\lambda \frac{\partial A_{2}}{\partial x_{2}} \dot{x}_{2},
\end{gathered}
$$

the Euler-Lagrange equations

$$
\frac{d}{d s}\left(\frac{\partial L^{*}}{\partial \dot{x}_{i}}\right)=\frac{\partial L^{*}}{\partial x_{i}}, \quad i=1,2
$$

become

$$
\begin{aligned}
& \lambda\left(\frac{\partial A_{1}}{\partial x_{2}}+\frac{\partial A_{2}}{\partial x_{1}}\right) \dot{x}_{1}=0 \\
& \lambda\left(\frac{\partial A_{1}}{\partial x_{2}}+\frac{\partial A_{2}}{\partial x_{1}}\right) \dot{x}_{2}=0 .
\end{aligned}
$$

Let $\lambda \neq 0$. If $\dot{x}_{1}=\dot{x}_{2}=0$, from the horizontality constraint

$$
\dot{t}=A_{1}(x) \dot{x}_{1}-A_{2}(x) \dot{x}_{2}
$$

it follows that $\dot{t}=0$ and hence the curve $\left(x_{1}(s), x_{2}(s), t(s)\right)$ degenerates to a point. Hence $\dot{x}_{1}$ and $\dot{x}_{2}$ do not vanish simultaneously and using that

$$
\Omega=\left(\frac{\partial A_{1}}{\partial x_{2}}+\frac{\partial A_{2}}{\partial x_{1}}\right) d x_{1} \wedge d x_{2}
$$

we have obtained the following result:

Proposition 7.1. The abnormal geodesics are horizontal curves $\gamma(s)$ with vanishing curvature

$$
\Omega(\gamma(s))=0
$$

REMARK 7.2. The one-form $\omega$ has the meaning of a magnetic potential, while the two form $\Omega=d \omega$ is the magnetic field. Then if the magnetic fields is vanishing 
along a horizontal direction, then the integral curve of that direction is an abnormal minimizer.

It is worthwhile to look at few particular cases.

- If $\theta=d t-2 x_{2} d x_{1}+2 x_{1} d x_{2}$, i.e., for $A_{1}(x)=2 x_{2}, A_{2}(x)=2 x_{1}$ the 2 -form of curvature never vanishes, $\Omega \neq 0$, so there are no abnormal geodesics in the Heisenberg case.

- In the case when $\theta=d t-e^{x_{2}} d x_{1}$ we also have no abnormal geodesics since $\Omega=d \theta \neq 0$.

- In the case of the Martinet distribution, which is defined by the Pfaff form $\theta=d t-\frac{1}{2}\left(x_{2}\right)^{2} d x_{1}$, we have that the abnormal geodesics are horizontal curves along which $x_{2}=0$. Hence $\dot{t}=0$ and hence the abnormal geodesics are given by $(s, 0, c)$, $c \in \mathbb{R}$, where we choose $x_{1}(s)=s$. For $c=0$ we obtain the abnormal minimizer quoted in Agrachev, Bonnai, Chuba and Kupka [1].

- The normal geodesics of the following model was first investigated in Calin [5] and later developed in Calin, Chang, Greiner [8]. Consider

$$
\omega=d t+k|x|^{2(k-1)}\left(x_{1} d x_{2}-x_{2} d x_{1}\right),
$$

where $k \in \mathbb{N}$, which defines the distribution spanned by the vector fields

$$
X_{1}=\partial_{x_{1}}+2 k x_{2}|x|^{2(k-1)} \partial_{t}, \quad X_{2}=\partial_{x_{2}}-2 k x_{1}|x|^{2(k-1)} \partial_{t} .
$$

Since $\Omega=d \omega=4 k^{2}|x|^{2(k-1)} d x_{1} \wedge d x_{2}$, it follows that the curvature form vanishes along the $t$-axis, where $x=0$. Since the $t$-axis is not a horizontal curve, it follows that in this case there are no abnormal geodesics.

REMARK 7.3. When $\ell_{0}=1$, we are dealing with only normal geodesics. In this case, we may also use Hamilton function to obtain the Euler-Lagrange system, i.e., the Hamiltonian formalism and Lagrange formalism are equivalent. Since $X_{1}=$ $\partial_{x_{1}}+A_{1}(x) \partial_{t}$ and $X_{2}=\partial_{x_{1}}-A_{2}(x) \partial_{t}$, the Hamiltonian for the operator $X_{1}^{2}+X_{2}^{2}$ is

$$
H=\frac{1}{2}\left[\left(\xi_{1}+A_{1}(x) \lambda\right)^{2}+\left(\xi_{2}-A_{2}(x) \lambda\right)^{2}\right] .
$$

The Hamiltonian system is

$$
\begin{aligned}
& \dot{x}_{1}=H_{\xi_{1}}=\xi_{1}+A_{1}(x) \lambda \\
& \dot{x}_{2}=H_{\xi_{2}}=\xi_{2}-A_{2}(x) \lambda \\
& \dot{\xi}_{1}=-H_{x_{1}}=\lambda\left(\frac{\partial A_{2}}{\partial x_{1}} \dot{x}_{2}-\frac{\partial A_{1}}{\partial x_{1}} \dot{x}_{1}\right) \\
& \dot{\xi}_{2}=-H_{x_{2}}=\lambda\left(\frac{\partial A_{2}}{\partial x_{2}} \dot{x}_{2}-\frac{\partial A_{1}}{\partial x_{2}} \dot{x}_{1}\right) .
\end{aligned}
$$

Differentiating the first two equations and substituting the last two yields the system (2.4)-(2.5) obtained by means of Lagrangian formalism.

\section{REFERENCES}

[1] A. Agrachev, B. Bonnaid, M. Chyba And I. Kupkia, Sub-Riemannian sphere in Martinet flat case, Control, Optimization and Calculus of Variations, 2 (1997), pp. 377-448. 
[2] R. Beals, B. Gaveau and P.C. Greiner, Hamilton-Jacobi Theory and the Heat Kernel on Heisenberg Groups, J. Math. Pures Appl., 79:7 (2000), pp. 633-689.

[3] G.A. Bliss, Lectures on Calculus of Variations, The University of Chicago Press, Chicago, 1946.

[4] O. Bolza, Lectures on Calculus of Variations, The University of Chicago Press, Chicago, 1964.

[5] O. Calin, Ph.D. Thesis, University of Toronto, Toronto, 2000.

[6] O. Calin, D.C. Chang and P.C. Greiner, On a step $2(k+1)$ subRiemannian manifold, J. Geometric Analysis, 14 (2004), pp. 1-18.

[7] O. Calin, D.C. Chang And P.C. Greiner, Real and complex Hamiltonian mechanics on some subRiemannian manifolds, Asian J. Math., 8 (2004), pp. 137-160.

[8] O. Calin, D.C. Chang And P.C. Greiner, Geometric Analysis on the Heisenberg Groups and Its Generalizations, AMS/IP Studies in Advanced Math. Series, 40, ISBN-10: 0-82184319-2, Amer. Math. Soc. and International Press, Boston, Massachusettes, (2007).

[9] O. Calin and D.C. Chang, Geometric Mechanics on Riemannian Manifolds, ISBN 0-81764354-0, Birkhäuser, Boston-Basel-Berlin, (2005).

[10] W.L. ChOw, Uber Systeme van Linearen Partiellen Differentialgleichungen erster Ordnung, Math. Ann., 117 (1939), pp. 98-105.

[11] P. Funk, Variationsrechnung und ihre Anwendung in Physik und Technik, Grundlehren der mathematischen Wissenschaften, bd., 94, Springer, Berlin•Heidelberg•New York, 2nd Edition, 1970.

[12] B. Gaveau, Principe de moindre action, propagation de la chaleur et estimees sous elliptiques sur certains groupes nilpotents, Acta Math., 139 (1977), pp. 95-153.

[13] B. Gaveau and P.C. Greiner, On geodesics in subRiemannian geometry, to appeared in Bulletin of Academia Sinica, (2006).

[14] M. Giaquinta And S. Hildebrandt, Calculus of Variations I, The Lagrangian Formalism, Grundlehren der mathematischen Wissenschaften, bd., 310, Springer,

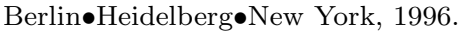

[15] P.C. Greiner and O. Calin, On subRiemannian geodesics, Anal. Appl., 1 (2003), pp. 289-350.

[16] L. Hörmander, Hypoelliptic Second Order Differential Equations, Acta Math., 119 (1975), pp. 141-171.

[17] D.F. Lawden, Elliptic functions and Applications, Applied Math. Sciences, 80, Springer-Verlag, New York, 1989.

[18] R. Millman and G. Parker, Elements of Differential Geometry, Prentice Hall, New York, 1977.

[19] H. Sagan, Introduction to Calculus of Variations, McGraw-Hill, New York, 1969.

[20] R. Strichartz, Subriemannian geometry, J. Diff. Geometry, 24 (1986), pp. 221-263; Correction, ibid., 30 (1989), pp. 595-596. 
O. CALIN ET. AL. 